\title{
New Oscillatory Theorems for Third-Order Nonlinear Delay Dynamic Equations on Time Scales
}

\author{
Li Gao', Shouhua Liu' ${ }^{1}$ Xiaotong Zheng \\ ${ }^{1}$ College of Science, Binzhou University, Shandong, China \\ ${ }^{2}$ School of Statistics, Renmin University of China, Beijing, China \\ Email: gaolibzxy@163.com, L3169207@163.com, \\ zxt22992299@163.com
}

How to cite this paper: Gao, L., Liu, S.H. and Zheng, X.T. (2018) New Oscillatory Theorems for Third-Order Nonlinear Delay Dynamic Equations on Time Scales. Journal of Applied Mathematics and Physics, 6, 232-246.

https://doi.org/10.4236/jamp.2018.61023

Received: November 27, 2017

Accepted: January 26, 2018

Published: January 29, 2018

Copyright $\odot 2018$ by authors and Scientific Research Publishing Inc. This work is licensed under the Creative Commons Attribution International License (CC BY 4.0).

http://creativecommons.org/licenses/by/4.0/

\section{Abstract}

This paper is concerned with the oscillatory properties of the third-order nonlinear delay dynamic equations of the form

$\left(r_{2}(t)\left[\left(r_{1}(t) x^{\Delta}(t)\right)^{\Delta}\right]^{\alpha}\right)^{\Delta}+q(t) f(x[\tau(t)])=0, \quad t \in \mathbb{T}$

on time scales $\mathbb{T}$, where $\alpha \geq 1$ is a quotient of odd positive integers. Applying the inequality technique we present two new sufficient conditions which ensure that every solution of equations is oscillatory or converges to zero. The results obtained improve and complement some known results in the literature.

\section{Keywords}

Oscillatory, Third Order, Delay Dynamic Equation, Generalized Riccati Transformation, Time Scale

\section{Introduction}

Beginning with the landmark contribution work of Hilger [1], the time scales theory, which in order to unify the continuous and discrete analysis, has received significant attention. In the recent years, there has been increasing interest in obtaining sufficient conditions for the oscillation and nonoscillation of solutions of various equations on time scales; we refer the reader to the papers [2]-[18]. Following this trend, we shall consider oscillation for the third-order nonlinear delay dynamic equation 


$$
\left(r_{2}(t)\left[\left(r_{1}(t) x^{\Delta}(t)\right)^{\Delta}\right]^{\alpha}\right)^{\Delta}+q(t) f(x[\tau(t)])=0, t \in \mathbb{T}, t \geq t_{0},
$$

where $\alpha \geq 1$ is a quotient of odd positive integers.

Throughout this paper, assume that

$\left(\mathrm{H}_{1}\right) \mathbb{T}$ is a time scale (i.e., a nonempty closed subset of the real numbers $\mathbb{R}$ ) which is unbounded above, and $t_{0} \in \mathbb{T}$ with $t_{0}>0$. We define the time scale interval of the form $\left[t_{0}, \infty\right)_{\mathbb{T}}$ by $\left[t_{0}, \infty\right)_{\mathbb{T}}=\left[t_{0}, \infty\right) \cap \mathbb{T}$.

$\left(\mathrm{H}_{2}\right) \quad r_{1}(t), r_{2}(t), q(t)$ are positive, real-valued rd-continuous functions defined on $\mathbb{T}$, and $r_{1}(t), r_{2}(t)$ satisfy

$$
\int_{t_{0}}^{\infty} \frac{1}{r_{1}(s)} \Delta s=\infty, \int_{t_{0}}^{\infty}\left(\frac{1}{r_{2}(s)}\right)^{\frac{1}{\alpha}} \Delta s=\infty .
$$

$\left(\mathrm{H}_{3}\right) \quad \tau: \mathbb{T} \rightarrow \mathbb{T}$ is a strictly increasing and differentiable function such that

$$
\tau(t) \leq t, \lim _{t \rightarrow \infty} \tau(t)=\infty \text { and } \tau(\mathbb{T})=\mathbb{T} .
$$

$\left(\mathrm{H}_{4}\right) \quad f: \mathbb{R} \rightarrow \mathbb{R}$ is a continuous function, and there exists a positive number $K$ such that $\frac{f(x)}{x^{\alpha}} \geq K>0$ for $x \neq 0$.

By a solution of (1), we mean a nontrivial function $x(t)$ satisfying (1) which has the properties $x(t) \in \mathrm{C}_{r d}^{1}\left(\left[T_{x}, \infty\right)_{\mathbb{T}}, \mathbb{R}\right)$ for $T_{x} \geq t_{0}$, and $r_{2}(t)\left[\left(r_{1}(t) x^{\Delta}(t)\right)^{\Delta}\right]^{\alpha} \in \mathrm{C}_{r d}^{1}\left(\left[T_{x}, \infty\right)_{\mathbb{T}}, \mathbb{R}\right)$. Our attention is restricted to those solutions of (1) which satisfy $\sup \{|x(t)|: t \geq T\}>0$ for all $T \geq T_{x}$. A solution $x$ of Equation (1) is said to be oscillatory on $\left[T_{x}, \infty\right)_{\mathbb{T}}$ if it is neither eventually positive nor eventually negative. Otherwise it is called nonoscillatory. The equation itself is called oscillatory if all its solutions are oscillatory.

If $\alpha=1, \tau(t)=t$, then (1) simplifies to the third-order nonlinear dynamic equation

$$
\left(r_{2}(t)\left[\left(r_{1}(t) x^{\Delta}(t)\right)^{\Delta}\right]\right)^{\Delta}+q(t) f(x(t))=0, t \in \mathbb{T}, t \geq t_{0} .
$$

If, furthermore, $r_{1}(t)=r_{2}(t)=1, f(x)=x, \tau(t)=t$, then (1) reduces to the third-order linear dynamic equation

$$
x^{\Delta \Delta \Delta}(t)+q(t) x(t)=0, t \in \mathbb{T}, t \geq t_{0} .
$$

If, in addition, $\alpha=1$, then (1) reduces to the nonlinear delay dynamic equation

$$
\left(r_{2}(t)\left[\left(r_{1}(t) x^{\Delta}(t)\right)^{\Delta}\right]\right)^{\Delta}+q(t) f(x[\tau(t)])=0, t \in \mathbb{T}, t \geq t_{0} .
$$

In [11], Erbe et al. established some sufficient conditions which ensure that every solution of Equation (2) is oscillatory or converges to zero. In [12], Erbe et al. studied the third-order linear dynamic Equation (3), and they obtained Hille and Nehari type oscillation criteria for the Equation (3). In [16], Han et al. 
extended and improved the results of [11] [12], meanwhile obtained some oscillatory criteria for the Equation (4). In [18], Gao et al. considered the third-order nonlinear dynamic Equation (1). By employing the generalized Riccati transformation and the integral averaging technique, they established three sufficient conditions which ensure that every solution of Equation (1) is oscillatory or converges to zero. On this basis, we continue to study Equation (1). If (4.11) in ([18], Theorem 4.3) is not hold, then we obtain two new sufficient conditions which guarantee that every solution of Equation (1) is oscillatory or converges to zero. Our results will improve some previous results. The usual notation and concepts of the time scales calculus, which will be used throughout the paper, can be found in [19] [20].

\section{Several Lemmas}

Lemma 1 Assume that $x(t)$ is an eventually positive solution of (1). Then there exists $T \in\left[t_{0}, \infty\right)_{\mathbb{T}}$ such that either

(I) $x(t)>0, x^{\Delta}(t)>0,\left(r_{1}(t) x^{\Delta}(t)\right)^{\Delta}>0, t \in[T, \infty)_{\mathbb{T}} ;$

or

(II) $x(t)>0, x^{\Delta}(t)<0,\left(r_{1}(t) x^{\Delta}(t)\right)^{\Delta}>0, t \in[T, \infty)_{\mathbb{T}}$.

The proof is similar to that of ([11], Lemma 1$)$.

Lemma 2 (see [19], Theorem 1.90]) If $x$ is differentiable, then

$$
\left(x^{\gamma}\right)^{\Delta}=\gamma x^{\Delta} \int_{0}^{1}\left[h x^{\sigma}+(1-h) x\right]^{\gamma-1} \mathrm{~d} h .
$$

Lemma 3 (see [21], Theorem 41]) Assume that $X$ and $Y$ are nonnegative real numbers. Then

$$
\lambda X Y^{\lambda-1}-X^{\lambda} \leq(\lambda-1) Y^{\lambda} \text { for all } \lambda>1,
$$

where the equality holds if and only if $X=Y$.

Throughout this paper, for sufficiently large $T$, we denote

$$
R(t, T)=\int_{T}^{t}\left(\frac{1}{r_{2}(s)}\right)^{\frac{1}{\alpha}} \Delta s .
$$

Lemma 4 Assume that $x(t)$ is an eventually positive solution of (1) which satisfies case (I) in Lemma 1 . Then there exists $T \in\left[t_{0}, \infty\right)_{\mathbb{T}}$, such that

$$
x^{\Delta}(t) \geq \frac{R(t, T)}{r_{1}(t)} r_{2}^{\frac{1}{\alpha}}(t)\left(r_{1}(t) x^{\Delta}(t)\right)^{\Delta}, t \in[T, \infty)_{\mathbb{T}} .
$$

The proof is similar to that of (18], Lemma 3.4).

Lemma 5 Assume that $x(t)$ is an eventually positive solution of (1) which satisfies case (I) in Lemma 1. Furthermore, assume that $r_{1}^{\Delta}(t) \leq 0$ and

$$
\int_{t_{0}}^{\infty} q(s) \tau^{\alpha}(s) \Delta s=\infty
$$


Then there exists $T \in\left[t_{0}, \infty\right)_{\mathbb{T}}$ such that $x(t)>t x^{\Delta}(t)$, and $\frac{x(t)}{t}$ is strictly decreasing on $[T, \infty)_{\mathbb{T}}$.

The proof is similar to that of ([16], Lemma 2.3).

Lemma 6 Assume that $x(t)$ is an eventually positive solution of (1) which satisfies case (II) in Lemma 1. Furthermore,

$$
\int_{t_{0}}^{\infty} \frac{1}{r_{1}(t)} \int_{t}^{\infty}\left[\frac{1}{r_{2}(s)} \int_{s}^{\infty} q(u) \Delta u\right]^{\frac{1}{\alpha}} \Delta s \Delta t=\infty .
$$

Then $\lim _{t \rightarrow \infty} x(t)=0$.

Proof Assume that $x(t)$ is an eventually positive solution of (1) which satisfies the case (II) in Lemma 1. Then $x(t)$ is decreasing and $\lim x(t)=l \geq 0$. We assert that $l=0$. If not, then $x[\tau(t)] \geq x(t) \geq l>0$ for $t \in\left[t_{0}, \infty\right)_{\mathbb{T}}$. Integrating (1) from $t$ to $\infty$, we get

$$
\begin{aligned}
& -r_{2}(t)\left[\left(r_{1}(t) x^{\Delta}(t)\right)^{\Delta}\right]^{\alpha} \\
& \leq-K \int_{t}^{\infty} q(s) x^{\alpha}[\tau(s)] \Delta s \leq-K l^{\alpha} \int_{t}^{\infty} q(s) \Delta s, t \in\left[t_{0}, \infty\right)_{\mathbb{T}} .
\end{aligned}
$$

Hence, we have

$$
-\left(r_{1}(t) x^{\Delta}(t)\right)^{\Delta} \leq-l\left[\frac{1}{r_{2}(t)} \int_{t}^{\infty} K q(s) \Delta s\right]^{\frac{1}{\alpha}} .
$$

Integrating the above inequality from $t$ to $\infty$, we obtain

$$
r_{1}(t) x^{\Delta}(t) \leq-l K^{\frac{1}{\alpha}} \int_{t}^{\infty}\left[\frac{1}{r_{2}(s)} \int_{s}^{\infty} q(u) \Delta u\right]^{\frac{1}{\alpha}} \Delta s .
$$

Integrating the last inequality again from $T$ to $t$, we have

$$
x(t)-x(T) \leq-I K^{\frac{1}{\alpha}} \int_{T}^{t} \frac{1}{r_{1}(s)} \int_{s}^{\infty}\left[\frac{1}{r_{2}(u)} \int_{u}^{\infty} q(v) \Delta v\right]^{\frac{1}{\alpha}} \Delta u \Delta s .
$$

Since condition (9) holds, we obtain $\lim _{t \rightarrow \infty} x(t)=-\infty$, which contradicts $x(t)>0$. Hence $l=0$. This completes the proof.

Lemma 7 (see [22], Theorem 3]) Let $a, b \in \mathbb{T}$ and $a<b$, for positive rd-continuous functions $f, g:[a, b] \rightarrow \mathbb{R}$, we have

$$
\int_{a}^{b}|f(s) g(s)| \Delta s \leq\left(\int_{a}^{b}|f(s)|^{p} \Delta s\right)^{\frac{1}{p}}\left(\int_{a}^{b}|g(s)|^{q} \Delta s\right)^{\frac{1}{a}},
$$

where $p>1$ and $\frac{1}{p}+\frac{1}{q}=1$.

\section{Main Results}

Theorem 1 Assume that (8) and (9) hold, $r_{1}^{\Delta}(t) \leq 0$. Furthermore, assume that 
there exist functions $H, h \in \mathrm{C}_{r d}(\mathbb{D}, \mathbb{R})$, where $\mathbb{D} \equiv\{(t, s): t \geq s \geq T\}$ such that

$$
H(t, t)=0, t \geq T ; H(t, s)>0, t>s \geq T,
$$

and $H$ has a nonpositive continuous $\Delta$-partial derivative $H^{\Delta_{s}}(t, s)$ with respect to the second variable and satisfies

$$
H^{\Delta_{s}}(\sigma(t), s)+\frac{\left(\delta^{\Delta}(s)\right)_{+}}{\delta(s)} H(\sigma(t), s)=-\frac{h(t, s)}{\delta(s)} H^{\frac{\alpha}{\alpha+1}}(\sigma(t), s),
$$

and, for all sufficiently large $T$, that there exists $T_{0} \geq T$,

$$
\begin{gathered}
0<\inf _{s \geq T_{0}}\left[\liminf _{t \rightarrow \infty} \frac{H(\sigma(t), s)}{H\left(\sigma(t), T_{0}\right)}\right] \leq \infty, \\
\limsup _{t \rightarrow \infty} \frac{1}{H\left(\sigma(t), T_{0}\right)} \int_{T_{0}}^{\sigma(t)} \frac{h_{-}^{\alpha+1}(t, s)\left[\sigma^{\alpha}(s) r_{1}(s)\right]^{\alpha}}{\left[s^{\alpha} \delta(\sigma(s)) R(s, T)\right]^{\alpha}} \Delta s<\infty,
\end{gathered}
$$

and a real rd-continuous function $\Psi:\left[t_{0}, \infty\right)_{\mathbb{T}} \rightarrow \mathbb{R}$ such that

$$
\begin{gathered}
\int_{T}^{\infty} \frac{s^{\alpha} \delta(\sigma(s)) R(s, T)}{\delta^{1+\frac{1}{\alpha}}(s) \sigma^{\alpha}(s) r_{1}(s)} \Psi_{+}^{1+\frac{1}{\alpha}}(s) \Delta s=\infty, \\
\limsup _{t \rightarrow \infty} \frac{1}{H\left(\sigma(t), T_{0}\right)} \int_{T_{0}}^{\sigma(t)}[H(\sigma(t), s) Q(s) \\
\left.-\frac{h_{-}^{\alpha+1}(t, s)\left[\sigma^{\alpha}(s) r_{1}(s)\right]^{\alpha}}{(\alpha+1)^{\alpha+1}\left[s^{\alpha} \delta(\sigma(s)) R(s, T)\right]^{\alpha}}\right] \Delta s \geq \Psi\left(T_{0}\right),
\end{gathered}
$$

where $\delta(t)$ is a positive $\Delta$-differentiable function,

$$
\begin{aligned}
& Q(t)=K q(t) \delta(\sigma(t))\left(\frac{\tau(t)}{\sigma(t)}\right)^{\alpha} . \\
& h_{-}(t, s)=\max \{0,-h(t, s)\}, h_{+}(t, s)=\max \{0, h(t, s)\}, \Psi_{+}(t)=\max \{0, \Psi(t)\} .
\end{aligned}
$$

Then every solution $x(t)$ of Equation (1) is either oscillatory or converges to zero.

Proof Assume that (1) has a nonoscillatory solution $x(t)$ on $\left[t_{0}, \infty\right)_{\mathbb{T}}$. Without loss generality we may assume that there exists sufficiently large $T \geq t_{0}$ such that $x(t)>0$ and $x[\tau(t)]>0$ for all $t \in[T, \infty)_{\mathbb{T}}$. By Lemma 1 , we see that $x(t)$ satisfies either case (I) or case (II).

If case (I) holds, then $x^{\Delta}(t)>0, t \in[T, \infty)_{\mathbb{T}}$. Define the function $\omega(t)$ by

$$
\omega(t)=\delta(t) r_{2}(t)\left(\frac{\left(r_{1}(t) x^{\Delta}(t)\right)^{\Delta}}{x(t)}\right)^{\alpha}, t \in[T, \infty)_{\mathbb{T}} .
$$

Obviously $\omega(t)>0$. Using the product and quotient rule of $\Delta$-differential, we obtain 


$$
\begin{aligned}
\omega^{\Delta}(t)= & \frac{\delta^{\Delta}(t)}{\delta(t)} \omega(t)-\delta(\sigma(t)) \frac{q(t) f(x[\tau(t)])}{x^{\alpha}(\sigma(t))} \\
& -\delta(\sigma(t)) r_{2}(t)\left[\frac{\left(r_{1}(t) x^{\Delta}(t)\right)^{\Delta}}{x(t)}\right]^{\alpha} \frac{\left(x^{\alpha}(t)\right)^{\Delta}}{x^{\alpha}(\sigma(t))} .
\end{aligned}
$$

By Lemma 2, we get

$$
\begin{aligned}
\omega^{\Delta}(t) \leq & \frac{\left(\delta^{\Delta}(t)\right)_{+}}{\delta(t)} \omega(t)-\delta(\sigma(t)) \frac{q(t) f(x[\tau(t)])}{x^{\alpha}(\sigma(t))} \\
& -\alpha \delta(\sigma(t)) r_{2}(t)\left[\frac{\left(r_{1}(t) x^{\Delta}(t)\right)^{\Delta}}{x(t)}\right]^{\alpha} \frac{x^{\alpha}(t) x^{\Delta}(t)}{x^{\alpha}(\sigma(t)) x(t)},
\end{aligned}
$$

where $\left(\delta^{\Delta}(t)\right)_{+}=\max \left\{0, \delta^{\Delta}(t)\right\}$, and get

$$
\begin{aligned}
\omega^{\Delta}(t) \leq & \frac{\left(\delta^{\Delta}(t)\right)_{+}}{\delta(t)} \omega(t)-\delta(\sigma(t)) \frac{q(t) f(x[\tau(t)])}{x^{\alpha}(\sigma(t))} \\
& -\alpha \delta(\sigma(t)) r_{2}(t)\left[\frac{\left(r_{1}(t) x^{\Delta}(t)\right)^{\Delta}}{x(t)}\right]^{\alpha} \frac{x^{\alpha}(t) x^{\Delta}(t)}{x^{\alpha}(\sigma(t)) x(t)} .
\end{aligned}
$$

From Lemma 5, we obtain $\frac{x(\tau(t))}{x(\sigma(t))} \geq \frac{\tau(t)}{\sigma(t)}, \frac{x(t)}{x(\sigma(t))} \geq \frac{t}{\sigma(t)}$, and using (7), so we obtain

$$
\begin{aligned}
\omega^{\Delta}(t) \leq & -K \delta(\sigma(t)) q(t)\left(\frac{\tau(t)}{\sigma(t)}\right)^{\alpha}+\frac{\left(\delta^{\Delta}(t)\right)_{+}}{\delta(t)} \omega(t) \\
& -\frac{\alpha \delta(\sigma(t)) R(t, T)}{r_{1}(t)}\left(\frac{t}{\sigma(t)}\right)^{\alpha} r_{2}^{1+\frac{1}{\alpha}}(t)\left[\frac{\left(r_{1}(t) x^{\Delta}(t)\right)^{\Delta}}{x(t)}\right]^{\alpha+1} .
\end{aligned}
$$

Hence, by the definition of $\omega(t), Q(t)$, we obtain

$$
\omega^{\Delta}(t) \leq-Q(t)+\frac{\left(\delta^{\Delta}(t)\right)_{+}}{\delta(t)} \omega(t)-\frac{\alpha \delta(\sigma(t)) R(t, T)}{\delta^{1+\frac{1}{\alpha}}(t) r_{1}(t)}\left(\frac{t}{\sigma(t)}\right)^{\alpha} \omega^{1+\frac{1}{\alpha}}(t)
$$

Multiplying both sides of (17), with $t$ replaced by $s$, by $H(\sigma(t), s)$, integrating with respect to $s$ from $T_{0}$ to $\sigma(t), t \geq T_{0} \geq T$, we get

$$
\begin{aligned}
& \int_{T_{0}}^{\sigma(t)} H(\sigma(t), s) Q(s) \Delta s \\
& \leq-\int_{T_{0}}^{\sigma(t)} H(\sigma(t), s) \omega^{\Delta}(s) \Delta s+\int_{T_{0}}^{\sigma(t)} \frac{H(\sigma(t), s)\left(\delta^{\Delta}(s)\right)_{+}}{\delta(s)} \omega(s) \Delta s \\
& -\int_{T_{0}}^{\sigma(t)} \frac{\alpha s^{\alpha} H(\sigma(t), s) \delta(\sigma(s)) R(s, T)}{\delta^{1+\frac{1}{\alpha}}} \omega^{1+\frac{1}{\alpha}}(s) \Delta s .
\end{aligned}
$$

Integrating by parts and using (11), we obtain 


$$
\begin{aligned}
& \int_{T_{0}}^{\sigma(t)} H(\sigma(t), s) Q(s) \Delta s \leq H\left(\sigma(t), T_{0}\right) \omega\left(T_{0}\right) \\
& +\int_{T_{0}}^{\sigma(t)} H^{\Delta_{s}}(\sigma(t), s) \omega(s) \Delta s+\int_{T_{0}}^{\sigma(t)} \frac{H(\sigma(t), s)\left(\delta^{\Delta}(s)\right)_{+}}{\delta(s)} \omega(s) \Delta s \\
& -\int_{T_{0}}^{\sigma(t)} \frac{\alpha s^{\alpha} H(\sigma(t), s) \delta(\sigma(s)) R(s, T)}{\delta^{1+\frac{1}{\alpha}}(s) \sigma^{\alpha}(s) r_{1}(s)} \omega^{1+\frac{1}{\alpha}}(s) \Delta s \\
& \leq H\left(\sigma(t), T_{0}\right) \omega\left(T_{0}\right)+\int_{T_{0}}^{\sigma(t)}\left[-\frac{h(t, s) H^{\frac{\alpha}{1+\alpha}}(\sigma(t), s)}{\delta(s)} \omega(s)\right. \\
& \left.-\frac{\alpha s^{\alpha} H(\sigma(t), s) \delta(\sigma(s)) R(s, T)}{\delta^{1+\frac{1}{\alpha}}(s) \sigma^{\alpha}(s) r_{1}(s)}\right] \Delta s,
\end{aligned}
$$

and so

$$
\begin{aligned}
& \int_{T_{0}}^{\sigma(t)} H(\sigma(t), s) Q(s) \Delta s \\
& \leq H\left(\sigma(t), T_{0}\right) \omega\left(T_{0}\right)+\int_{T_{0}}^{\sigma(t)}\left[-\frac{h(t, s) H^{\frac{\alpha}{1+\alpha}}(\sigma(t), s)}{\delta(s)} \omega(s)\right. \\
& \left.-\frac{\alpha s^{\alpha} H(\sigma(t), s) \delta(\sigma(s)) R(s, T)}{\delta^{1+\frac{1}{\alpha}}(s) \sigma^{\alpha}(s) r_{1}(s)} \omega^{1+\frac{1}{\alpha}}(s)\right] \Delta s,
\end{aligned}
$$

Now set

$$
\begin{gathered}
X^{\lambda}=\frac{\alpha s^{\alpha} H(\sigma(t), s) \delta(\sigma(s)) R(s, T)}{\delta^{1+\frac{1}{\alpha}}(s) \sigma^{\alpha}(s) r_{1}(s)} \omega^{\lambda}(s), \\
Y^{\lambda-1}=\frac{h_{-}(t, s)\left[\sigma^{\alpha}(s) r_{1}(s)\right]^{\frac{1}{\lambda}}}{\lambda\left[\alpha s^{\alpha} \delta(s) R(s, T)\right]^{\frac{1}{\lambda}}},
\end{gathered}
$$

where $\lambda=\frac{\alpha+1}{\alpha}>1, \quad X \geq 0$ and $Y \geq 0$. Using the inequality (6), we obtain

$$
\begin{aligned}
& \frac{h_{-}(t, s) H^{\frac{1}{\lambda}}(\sigma(t), s)}{\delta(s)} \omega(s)-\frac{\alpha s^{\alpha} H(\sigma(t), s) \delta(\sigma(s)) R(s, T)}{\delta^{\lambda-1}(s) \sigma^{\alpha}(s) r_{1}(s)} \omega^{\lambda}(s) \\
& \leq \frac{h_{-}^{\alpha+1}(t, s)\left[\sigma^{\alpha}(s) r_{1}(s)\right]^{\alpha}}{(\alpha+1)^{\alpha+1}\left[s^{\alpha} \delta(\sigma(s)) R(s, T)\right]^{\alpha}} .
\end{aligned}
$$

Combining (18) and (19), we get

$$
\begin{aligned}
& \frac{1}{H\left(\sigma(t), T_{0}\right)} \int_{T_{0}}^{\sigma(t)}\left[H(\sigma(t), s) Q(s)-\frac{h_{-}^{\alpha+1}(t, s)\left[\sigma^{\alpha}(s) r_{1}(s)\right]^{\alpha}}{(\alpha+1)^{\alpha+1}\left[s^{\alpha} \delta(\sigma(s)) R(s, T)\right]^{\alpha}}\right] \Delta s \\
& \leq \omega\left(T_{0}\right) .
\end{aligned}
$$


From (16), we obtain

$$
\begin{gathered}
\Psi\left(T_{0}\right) \leq \omega\left(T_{0}\right), T_{0} \in[T, \infty)_{\mathbb{T}}, \\
\limsup _{t \rightarrow \infty} \frac{1}{H\left(\sigma(t), T_{0}\right)} \int_{T_{0}}^{\sigma(t)} H(\sigma(t), s) Q(s) \Delta s \geq \Psi\left(T_{0}\right) .
\end{gathered}
$$

By (18), we get

$$
\begin{aligned}
& \frac{1}{H\left(\sigma(t), T_{0}\right)} \int_{T_{0}}^{\sigma(t)} H(\sigma(t), s) Q(s) \Delta s \\
& \leq \omega\left(T_{0}\right)+\frac{1}{H\left(\sigma(t), T_{0}\right)} \int_{T_{0}}^{\sigma(t)} \frac{h_{-}(t, s) H^{\frac{\alpha}{1+\alpha}}(\sigma(t), s)}{\delta(s)} \omega(s) \Delta s \\
& -\frac{1}{H\left(\sigma(t), T_{0}\right)} \int_{T_{0}}^{\sigma(t)} \frac{\alpha s^{\alpha} H(\sigma(t), s) \delta(\sigma(s)) R(s, T)}{\delta^{1+\frac{1}{\alpha}}(s) \sigma^{\alpha}(s) r_{1}(s)} \omega^{1+\frac{1}{\alpha}}(s) \Delta s .
\end{aligned}
$$

We denote

$$
\begin{gathered}
u(t)=\frac{1}{H\left(\sigma(t), T_{0}\right)} \int_{T_{0}}^{\sigma(t)} \frac{h_{-}(t, s) H^{\frac{\alpha}{1+\alpha}}(\sigma(t), s)}{\delta(s)} \omega(s) \Delta s, \\
v(t)=\frac{1}{H\left(\sigma(t), T_{0}\right)} \int_{T_{0}}^{\sigma(t)} \frac{\alpha s^{\alpha} H(\sigma(t), s) \delta(\sigma(s)) R(s, T)}{\delta^{1+\frac{1}{\alpha}}(s) \sigma^{\alpha}(s) r_{1}(s)} \omega^{1+\frac{1}{\alpha}}(s) \Delta s,
\end{gathered}
$$

meanwhile noting that (16), we obtain

$$
\liminf _{t \rightarrow \infty}[v(t)-u(t)] \leq \omega\left(T_{0}\right)-\Psi\left(T_{0}\right)<\infty .
$$

Now we assert that

$$
\int_{T}^{\infty} \frac{s^{\alpha} \delta(\sigma(s)) R(s, T)}{\delta^{1+\frac{1}{\alpha}}(s) \sigma^{\alpha}(s) r_{1}(s)} \omega^{1+\frac{1}{\alpha}}(s) \Delta s<\infty
$$

holds. Suppose to the contrary that

$$
\int_{T}^{\infty} \frac{s^{\alpha} \delta(\sigma(s)) R(s, T)}{\delta^{1+\frac{1}{\alpha}}(s) \sigma^{\alpha}(s) r_{1}(s)} \omega^{1+\frac{1}{\alpha}}(s) \Delta s=\infty
$$

by (13), there exists a constant $\varepsilon>0$ such that

$$
\inf _{s \geq T_{0}}\left[\liminf _{t \rightarrow \infty} \frac{H(\sigma(t), s)}{H\left(\sigma(t), T_{0}\right)}\right]>\varepsilon>0,
$$

from (23), there exists $T_{1} \in\left[T_{0}, \infty\right)_{\mathbb{T}}$ for arbitrary real number $M>0$ such that

$$
\int_{T_{1}}^{\sigma(t)} \frac{s^{\alpha} \delta(\sigma(s)) R(s, T)}{\delta^{1+\frac{1}{\alpha}}(s) \sigma^{\alpha}(s) r_{1}(s)} \omega^{1+\frac{1}{\alpha}}(s) \Delta s \geq \frac{M}{\alpha \varepsilon}, \text { for } t \in\left[T_{1}, \infty\right)_{\mathbb{T}}
$$

Using the integration by parts formula of $\Delta$-differential, we obtain 


$$
\begin{aligned}
v(t) & =\frac{1}{H\left(\sigma(t), T_{0}\right)} \int_{T_{0}}^{\sigma(t)}\left\{\alpha H(\sigma(t), s)\left(\int_{T_{0}}^{s} \frac{u^{\alpha} \delta(\sigma(u)) R(u, T)}{\delta^{1+\frac{1}{\alpha}}(u) \sigma^{\alpha}(u) r_{1}(u)} W^{1+\frac{1}{\alpha}}(u) \Delta u\right)^{\Delta_{s}}\right\} \Delta s \\
& =\frac{1}{H\left(\sigma(t), T_{0}\right)} \int_{T_{0}}^{\sigma(t)}\left\{-\alpha H^{\Delta_{s}}(\sigma(t), s) \int_{T_{0}}^{\sigma(s)} \frac{u^{\alpha} \delta(\sigma(u)) R(u, T)}{\delta^{1+\frac{1}{\alpha}}(u) \sigma^{\alpha}(u) r_{1}(u)} \omega^{1+\frac{1}{\alpha}}(u) \Delta u\right\} \Delta s \\
& \geq \frac{1}{H\left(\sigma(t), T_{0}\right)} \int_{T_{1}}^{\sigma(t)}\left\{-\alpha H^{\Delta_{s}}(\sigma(t), s) \int_{T_{0}}^{\sigma(s)} \frac{u^{\alpha} \delta(\sigma(u)) R(u, T)}{\delta^{1+\frac{1}{\alpha}}(u) \sigma^{\alpha}(u) r_{1}(u)} \omega^{1+\frac{1}{\alpha}}(u) \Delta u\right\} \Delta s \\
& \geq \frac{1}{H\left(\sigma(t), T_{0}\right)} \int_{T_{1}}^{\sigma(t)}-\alpha H^{\Delta_{s}}(\sigma(t), s) \frac{M}{\alpha \varepsilon} \Delta s=\frac{M}{\varepsilon} \frac{H\left(\sigma(t), T_{1}\right)}{H\left(\sigma(t), T_{0}\right)} .
\end{aligned}
$$

From (24), there exists $T_{2} \in\left[T_{1}, \infty\right)_{\mathbb{T}}$, we get $\frac{H\left(\sigma(t), T_{1}\right)}{H\left(\sigma(t), T_{0}\right)} \geq \varepsilon$ for $t \in\left[T_{2}, \infty\right)_{\mathbb{T}}$, so that $v(t) \geq M$. Since $M$ is arbitrary, we obtain

$$
\lim _{t \rightarrow \infty} v(t)=\infty .
$$

Selecting a sequence $\left\{t_{n}\right\}_{n=1}^{\infty}: t_{n} \in\left[T_{0}, \infty\right)_{\mathbb{T}}$ with $\lim _{n \rightarrow \infty} t_{n}=\infty$ satisfying

$$
\lim _{n \rightarrow \infty}\left[v\left(t_{n}\right)-u\left(t_{n}\right)\right]=\liminf _{t \rightarrow \infty}[v(t)-u(t)]<\infty,
$$

then there exists a constant $M_{0}>0$ such that

$$
v\left(t_{n}\right)-u\left(t_{n}\right) \leq M_{0}
$$

for sufficiently large positive integer $n$. From (22), we can easily see

$$
\lim _{n \rightarrow \infty} v\left(t_{n}\right)=\infty
$$

(26) implies that

$$
\lim _{n \rightarrow \infty} u\left(t_{n}\right)=\infty
$$

From (26) and (27), we obtain

$$
\frac{u\left(t_{n}\right)}{v\left(t_{n}\right)}-1 \geq-\frac{M_{0}}{v\left(t_{n}\right)}>-\frac{M_{0}}{2 M_{0}}=-\frac{1}{2},
$$

i.e.,

$$
\frac{u\left(t_{n}\right)}{v\left(t_{n}\right)}>\frac{1}{2}
$$

for sufficiently large positive integer $n$, which together with (28) implies

$$
\lim _{n \rightarrow \infty} \frac{\left[u\left(t_{n}\right)\right]^{\alpha+1}}{\left[v\left(t_{n}\right)\right]^{\alpha}}=\lim _{n \rightarrow \infty}\left[\frac{u\left(t_{n}\right)}{v\left(t_{n}\right)}\right]^{\alpha} u\left(t_{n}\right)=\infty .
$$

On the other hand, by Lemma 7, we obtain 


$$
\begin{aligned}
& u\left(t_{n}\right)=\frac{1}{H\left(\sigma\left(t_{n}\right), T_{0}\right)} \int_{T_{0}}^{\sigma\left(t_{n}\right)} \frac{h_{-}\left(t_{n}, s\right) H^{\frac{\alpha}{\alpha+1}}\left(\sigma\left(t_{n}\right), s\right)}{\delta(s)} \omega(s) \Delta s \\
& =\int_{T_{0}}^{\sigma\left(t_{n}\right)} \frac{h_{-}\left(t_{n}, s\right) H^{\frac{\alpha}{\alpha+1}}\left(\sigma\left(t_{n}\right), s\right)}{H\left(\sigma\left(t_{n}\right), T_{0}\right) \delta(s)} \omega(s) \Delta s \\
& =\int_{T_{0}}^{\sigma\left(t_{n}\right)}\left\{\left[\frac{\alpha s^{\alpha} H\left(\sigma\left(t_{n}\right), s\right) \delta(\sigma(s)) R(s, T)}{H\left(\sigma\left(t_{n}\right), T_{0}\right) \sigma^{\alpha}(s) r_{1}(s)}\right]^{\frac{\alpha}{\alpha+1}} \frac{\omega(s)}{\delta(s)}\right\} \\
& \times\left\{\frac{h_{-}\left(t_{n}, s\right) H^{\frac{\alpha}{\alpha+1}}\left(\sigma\left(t_{n}\right), s\right)}{H\left(\sigma\left(t_{n}\right), T_{0}\right)}\left[\frac{\alpha s^{\alpha} H\left(\sigma\left(t_{n}\right), s\right) \delta(\sigma(s)) R(s, T)}{H\left(\sigma\left(t_{n}\right), T_{0}\right) \sigma^{\alpha}(s) r_{1}(s)}\right]^{-\frac{\alpha}{\alpha+1}}\right\} \Delta s \\
& \leq\left\{\int_{T_{0}}^{\sigma\left(t_{n}\right)} \frac{\alpha s^{\alpha} H\left(\sigma\left(t_{n}\right), s\right) \delta(\sigma(s)) R(s, T)}{H\left(\sigma\left(t_{n}\right), T_{0}\right) \sigma^{\alpha}(s) r_{1}(s)}\left[\frac{\omega(s)}{\delta(s)}\right]^{\frac{\alpha+1}{\alpha}} \Delta s\right\}^{\frac{\alpha}{\alpha+1}} \\
& \times\left\{\int_{T_{0}}^{\sigma\left(t_{n}\right)} \frac{h_{-}\left(t_{n}, s\right) H^{\frac{\alpha}{\alpha+1}}\left(\sigma\left(t_{n}\right), s\right)}{H^{\alpha+1}\left(\sigma\left(t_{n}\right), T_{0}\right)}\left[\frac{\alpha s^{\alpha} H\left(\sigma\left(t_{n}\right), s\right) \delta(\sigma(s)) R(s, T)}{H\left(\sigma\left(t_{n}\right), T_{0}\right) \sigma^{\alpha}(s) r_{1}(s)}\right]^{-\alpha} \Delta s\right\}^{\frac{1}{\alpha+1}} \\
& =\left[v\left(t_{n}\right)\right]^{\frac{\alpha}{\alpha+1}}\left\{\frac{1}{\alpha^{\alpha} H\left(\sigma\left(t_{n}\right), T_{0}\right)} \int_{T_{0}}^{\sigma\left(t_{n}\right)} \frac{h_{-}^{\alpha+1}\left(t_{n}, s\right) \sigma^{\alpha^{2}}(s) r_{1}^{\alpha}(s)}{\left[s^{\alpha} \delta(\sigma(s)) R(s, T)\right]^{\alpha}} \Delta s\right\}^{\frac{1}{\alpha+1}} .
\end{aligned}
$$

The above inequality show that

$$
\frac{\left[u\left(t_{n}\right)\right]^{\alpha+1}}{\left[v\left(t_{n}\right)\right]^{\alpha}} \leq \frac{1}{\alpha^{\alpha} H\left(\sigma\left(t_{n}\right), T_{0}\right)} \int_{T_{0}}^{\sigma\left(t_{n}\right)} \frac{h_{-}^{\alpha+1}\left(t_{n}, s\right) \sigma^{\alpha^{2}}(s) r_{1}^{\alpha}(s)}{\left[s^{\alpha} \delta(\sigma(s)) R(s, T)\right]^{\alpha}} \Delta s .
$$

Hence, (29) implies

$$
\lim _{n \rightarrow \infty} \frac{1}{\alpha^{\alpha} H\left(\sigma\left(t_{n}\right), T_{0}\right)} \int_{T_{0}}^{\sigma\left(t_{n}\right)} \frac{h_{-}^{\alpha+1}\left(t_{n}, s\right) \sigma^{\alpha^{2}}(s) r_{1}^{\alpha}(s)}{\left[s^{\alpha} \delta(\sigma(s)) R(s, T)\right]^{\alpha}} \Delta s=\infty .
$$

This contradicts (14). Therefore (22) holds. Noting $\Psi\left(T_{0}\right) \leq \omega\left(T_{0}\right)$ for $T_{0} \in[T, \infty)_{\mathbb{T}}$, by using $(22)$, we obtain

$$
\int_{T}^{\infty} \frac{s^{\alpha} \delta(\sigma(s)) R(s, T)}{\delta^{1+\frac{1}{\alpha}}(s) \sigma^{\alpha}(s) r_{1}(s)} \Psi_{+}^{1+\frac{1}{\alpha}}(s) \Delta s \leq \int_{T}^{\infty} \frac{s^{\alpha} \delta(\sigma(s)) R(s, T)}{\delta^{1+\frac{1}{\alpha}}(s) \sigma^{\alpha}(s) r_{1}(s)} \omega^{1+\frac{1}{\alpha}}(s) \Delta s<\infty .
$$

This contradicts (15).

If case (II) holds, from (9), by Lemma $6 \lim _{t \rightarrow \infty} x(t)=0$. The proof is complete.

Theorem 2 Assume that (8), (9), (12), (13), (15) and $r_{1}^{\Delta}(t) \leq 0$ hold, where $H, h$ and $\delta$ are defined in Theorem 1. Furthermore, assume that there is a real rd-continuous function $\Psi:\left[t_{0}, \infty\right)_{\mathbb{T}} \rightarrow \mathbb{R}$ such that

$$
\liminf _{t \rightarrow \infty} \frac{1}{H\left(\sigma(t), T_{0}\right)} \int_{T_{0}}^{\sigma(t)} H(\sigma(t), s) Q(s) \Delta s<\infty,
$$




$$
\begin{aligned}
& \liminf _{t \rightarrow \infty} \frac{1}{H\left(\sigma(t), T_{0}\right)} \int_{T_{0}}^{\sigma(t)}[H(\sigma(t), s) Q(s) \\
& -\frac{h_{-}^{\alpha+1}(t, s)\left[\sigma^{\alpha}(s) r_{1}(s)\right]^{\alpha}}{(\alpha+1)^{\alpha+1}\left[s^{\alpha} \delta(\sigma(s)) R(s, T)\right]^{\alpha}} \Delta s \geq \Psi\left(T_{0}\right),
\end{aligned}
$$

for $T_{0} \in[T, \infty)_{\mathbb{T}}$, where $Q(t), \Psi(t)$ are defined in Theorem 1 . Then every solution $x(t)$ of Equation (1) is either oscillatory or converges to zero.

Proof Assume that (1) has a nonoscillatory solution $x(t)$ on $\left[t_{0}, \infty\right)_{\mathbb{T}}$. Without loss generality we may assume that there exists sufficiently large $T \geq t_{0}$ such that $x(t)>0$ and $x[\tau(t)]>0$ for all $t \in[T, \infty)_{\mathbb{T}}$. By Lemma 1, we see that $x(t)$ satisfies either case (I) or case (II).

If case (I) holds, proceeding as in the proof of Theorem 1, we get

$$
\begin{aligned}
& \frac{1}{H\left(\sigma(t), T_{0}\right)} \int_{T_{0}}^{\sigma(t)}[H(\sigma(t), s) Q(s) \\
& \left.-\frac{h_{-}^{\alpha+1}(t, s)\left[\sigma^{\alpha}(s) r_{1}(s)\right]^{\alpha}}{(\alpha+1)^{\alpha+1}\left[s^{\alpha} \delta(\sigma(s)) R(s, T)\right]^{\alpha}}\right] \Delta s \leq \omega\left(T_{0}\right),
\end{aligned}
$$

From (31), we obtain

$$
\begin{gathered}
\Psi\left(T_{0}\right) \leq \omega\left(T_{0}\right), T_{0} \in[T, \infty)_{\mathbb{T}} ; \\
\liminf _{t \rightarrow \infty} \frac{1}{H\left(\sigma(t), T_{0}\right)} \int_{T_{0}}^{\sigma(t)} H(\sigma(t), s) Q(s) \Delta s \geq \Psi\left(T_{0}\right) ;
\end{gathered}
$$

and

$$
\begin{aligned}
& \liminf _{t \rightarrow \infty} \frac{1}{H\left(\sigma(t), T_{0}\right)} \int_{T_{0}}^{\sigma(t)} H(\sigma(t), s) Q(s) \Delta s \\
& -\liminf _{t \rightarrow \infty} \frac{1}{H\left(\sigma(t), T_{0}\right)} \int_{T_{0}}^{\sigma(t)} \frac{h_{-}^{\alpha+1}(t, s)\left[\sigma^{\alpha}(s) r_{1}(s)\right]^{\alpha}}{(\alpha+1)^{\alpha+1}\left[s^{\alpha} \delta(\sigma(s)) R(s, T)\right]^{\alpha}} \Delta s \\
& \geq \liminf _{t \rightarrow \infty} \frac{1}{H\left(\sigma(t), T_{0}\right)} \int_{T_{0}}^{\sigma(t)}[H(\sigma(t), s) Q(s) \\
& -\frac{h_{-}^{\alpha+1}(t, s)\left[\sigma^{\alpha}(s) r_{1}(s)\right]^{\alpha}}{\left.(\alpha+1)^{\alpha+1}\left[s^{\alpha} \delta(\sigma(s)) R(s, T)\right]^{\alpha}\right] \Delta s} \\
& \geq \Psi\left(T_{0}\right) .
\end{aligned}
$$

Using (30) and (33), we get

$$
\liminf _{t \rightarrow \infty} \frac{1}{H\left(\sigma(t), T_{0}\right)} \int_{T_{0}}^{\sigma(t)} \frac{h_{-}^{\alpha+1}(t, s)\left[\sigma^{\alpha}(s) r_{1}(s)\right]^{\alpha}}{(\alpha+1)^{\alpha+1}\left[s^{\alpha} \delta(\sigma(s)) R(s, T)\right]^{\alpha}} \Delta s<\infty .
$$

Thus, there exists a sequence $\left\{t_{n}\right\}_{n=1}^{\infty}: t_{n} \in\left[T_{0}, \infty\right)_{\mathbb{T}}$ with $\lim _{n \rightarrow \infty} t_{n}=\infty$ such that 


$$
\lim _{n \rightarrow \infty} \frac{1}{H\left(\sigma\left(t_{n}\right), T_{0}\right)} \int_{T_{0}}^{\sigma\left(t_{n}\right)} \frac{h_{-}^{\alpha+1}(t, s)\left[\sigma^{\alpha}(s) r_{1}(s)\right]^{\alpha}}{(\alpha+1)^{\alpha+1}\left[s^{\alpha} \delta(\sigma(s)) R(s, T)\right]^{\alpha}} \Delta s<\infty .
$$

We define $u(t)$ and $v(t)$ also, as in the proof of Theorem 1. From (18) and (32), we obtain

$$
\limsup _{t \rightarrow \infty}[v(t)-u(t)] \leq \omega\left(T_{0}\right)-\liminf _{t \rightarrow \infty} \frac{1}{H\left(\sigma(t), T_{0}\right)} \int_{T_{0}}^{\sigma(t)} H(\sigma(t), s) Q(s) \Delta s<\infty .
$$

For the above sequence $\left\{t_{n}\right\}_{n=1}^{\infty}$, we get

$$
\lim _{n \rightarrow \infty}\left[v\left(t_{n}\right)-u\left(t_{n}\right)\right] \leq \limsup _{t \rightarrow \infty}[v(t)-u(t)]<\infty .
$$

Similar to the proof of Theorem 1, we get (22). The rest of the proof is similar to that of Theorem 1, and hence is omitted. The proof is complete.

Remark 1 From Theorems 1 and 2, we can obtain different sufficient conditions for the oscillation of Equation (1) with different choices of the functions $\delta$ and $H$. For example, $H(t, s)=(t-s)^{m}$ or $H(t, s)=\left(\ln \frac{t+1}{s+1}\right)^{m}$.

Remark 2 The theorems in this paper are new even for the cases of $\mathbb{T}=\mathbb{R}$ and $\mathbb{T}=\mathbb{Z}$.

Example 1 Consider the third-order nonlinear delay dynamic equation

$$
\left(t\left(\left(\frac{1}{t^{2}} x^{\Delta}(t)\right)^{\Delta}\right)^{\frac{5}{3}}\right)^{\Delta}+\frac{1}{t^{\frac{8}{3}}}\left(x\left(\frac{t}{2}\right)\right)^{\frac{5}{3}}\left(1+\ln \left(1+x^{2}\left(\frac{t}{2}\right)\right)\right)=0, t \in \overline{2^{\mathrm{Z}}}, t \geq t_{0}:=2 \text {. }
$$

Here $\quad \alpha=\frac{5}{3}, \quad r_{1}(t)=\frac{1}{t^{2}}, \quad r_{2}(t)=t, \quad q(t)=\frac{1}{t^{\frac{8}{3}}}, \quad f(x)=x^{\frac{5}{3}}\left(1+\ln \left(1+x^{2}\right)\right)$ and $\tau(t)=\frac{t}{2}<t$

The conditions $\left(\mathrm{H}_{1}\right)-\left(\mathrm{H}_{3}\right)$ are clearly satisfied, $\left(\mathrm{H}_{4}\right)$ holds with $K=1$. $\frac{f(x)}{x^{\alpha}}=1+\ln \left(1+x^{2}\right) \geq K>0 . r_{1}^{\Delta}(t)=-\frac{3}{4 t^{3}}<0$, and

$$
\begin{aligned}
\int_{t_{0}}^{\infty} q(s) & \tau^{\alpha}(s) \Delta s=\int_{2}^{\infty} \frac{1}{s^{\frac{8}{3}}}\left(\frac{s}{2}\right)^{\frac{5}{3}} \Delta s=2^{-\frac{5}{3}} \int_{2}^{\infty} s^{-1} \Delta s=\infty, \\
& \int_{t_{0}}^{\infty} \frac{1}{r_{1}(t)} \int_{t}^{\infty}\left[\frac{1}{r_{2}(s)} \int_{s}^{\infty} q(u) \Delta u\right]^{\frac{1}{\alpha}} \Delta s \Delta t \\
& =\int_{2}^{\infty} t^{2} \int_{t}^{\infty}\left[\frac{1}{s} \int_{s}^{\infty} \frac{1}{u^{\frac{8}{3}}} \Delta u\right]^{\frac{3}{5}} \Delta s \Delta t \\
& =\left(\frac{1}{1-2^{-\frac{5}{3}}}\right)^{\frac{3}{5}} \int_{2}^{\infty} t^{2} \int_{t}^{\infty} \frac{1}{s^{\frac{8}{5}}} \Delta s \Delta t \\
& =\left(\frac{1}{1-2^{-\frac{5}{3}}}\right)^{\frac{3}{5}} \frac{1}{1-2^{-\frac{3}{5}}} \int_{2}^{\infty} t^{\frac{7}{5}} \Delta t=\infty
\end{aligned}
$$


so (8), (9) hold. For larger enough $t>T$, we have

$$
R(t, T)=\int_{T}^{t}\left(\frac{1}{r_{2}(s)}\right)^{\frac{1}{\alpha}} \Delta s=\int_{T}^{t} s^{-\frac{3}{5}} \Delta s=\frac{t^{\frac{2}{5}}-T^{\frac{2}{5}}}{2^{\frac{2}{5}}-1}>1 .
$$

Let $\delta(t)=t$, since $\sigma(t)=2 t$, we have

$$
Q(t)=K q(t) \delta(\sigma(t))\left(\frac{\tau(t)}{\sigma(t)}\right)^{\alpha}=\frac{1}{2^{\frac{7}{3}} t^{\frac{5}{3}}} .
$$

Let $H(t, s)=(t-s)^{2}$, that there exists a function $h(t, s)=-\frac{4(t-s)^{2}}{(2 t-s)^{\frac{5}{4}}}$ such that

$$
H^{\Delta_{s}}(\sigma(t), s)+\frac{\left(\delta^{\Delta}(s)\right)_{+}}{\delta(s)} H(\sigma(t), s)=-\frac{h(t, s)}{\delta(s)} H^{\frac{\alpha}{\alpha+1}}(\sigma(t), s) .
$$

It follows that

$$
\begin{gathered}
0<\inf _{s \geq T_{0}}\left[\liminf _{t \rightarrow \infty} \frac{H(\sigma(t), s)}{H\left(\sigma(t), T_{0}\right)}\right]=\inf _{s \geq T_{0}}\left[\liminf _{t \rightarrow \infty} \frac{(2 t-s)^{2}}{\left(2 t-T_{0}\right)^{2}}\right]=1<\infty, \\
\limsup _{t \rightarrow \infty} \frac{1}{H\left(\sigma(t), T_{0}\right)} \int_{T_{0}}^{\sigma(t)} \frac{h_{-}^{\alpha+1}(t, s)\left[\sigma^{\alpha}(s) r_{1}(s)\right]^{\alpha}}{\left[s^{\alpha} \delta(\sigma(s)) R(s, T)\right]^{\alpha}} \Delta s \leq \frac{2^{\frac{10}{9}} \cdot T_{0}^{-4}}{1-2^{-4}}<\frac{2^{6}}{T_{0}^{4}}<\infty,
\end{gathered}
$$

so (12), (13) and (14) hold. Let $\Psi(t)=\frac{1}{2 t}$, we have

$$
\int_{T}^{\infty} \frac{s^{\alpha} \delta(\sigma(s)) R(s, T)}{\delta^{1+\frac{1}{\alpha}}(s) \sigma^{\alpha}(s) r_{1}(s)} \Psi_{+}^{1+\frac{1}{\alpha}}(s) \Delta s=\frac{1}{\left.2^{\frac{49}{15}} \cdot 2^{\frac{2}{5}}-1\right)} \cdot \int_{T}^{\infty}\left(s^{\frac{1}{5}}-s^{-\frac{1}{5}} \cdot T^{\frac{2}{5}}\right) \Delta s=\infty
$$

and

$$
\begin{aligned}
& \limsup _{t \rightarrow \infty} \frac{1}{H\left(\sigma(t), T_{0}\right)} \int_{T_{0}}^{\sigma(t)}\left[H(\sigma(t), s) Q(s)-\frac{h_{-}^{\alpha+1}(t, s)\left[\sigma^{\alpha}(s) r_{1}(s)\right]^{\alpha}}{(\alpha+1)^{\alpha+1}\left[s^{\alpha} \delta(\sigma(s)) R(s, T)\right]^{\alpha}}\right] \Delta s \\
& \geq \frac{2^{-\frac{7}{3}}}{\left(1-2^{-\frac{2}{3}}\right) T_{0}^{\frac{2}{3}}}-\frac{2^{\frac{10}{9}}}{\left(\frac{8}{3}\right)^{\frac{8}{3}}\left(1-2^{-4}\right) T_{0}^{4}}>\frac{1}{2 T_{0}^{\frac{2}{3}}}>\frac{1}{2 T_{0}}=\Psi\left(T_{0}\right) .
\end{aligned}
$$

Then, by Theorem 1, every solution $x(t)$ of Equation (34) is either oscillatory or converges to zero. But the results in [18] cannot be applied in (34).

\section{Acknowledgements}

The authors are grateful to the reviewers for their comments and suggestions. This research is supported by Shandong Provincial Natural Science Foundation (ZR2013AM003), Technology Planning Program of Shandong Province (J14LI54). 


\section{References}

[1] Hilger, S. (1990) Analysis on Measure Chains-A Unified Approach to Continuous and Discrete Calculus. Results in Mathematics, 18, 18-56.

https://doi.org/10.1007/BF03323153

[2] Bohner, M. and Saker, S.H. (2004) Oscillation of Second Order Nonlinear Dynamic Equations on Time Scales. Rocky Mountain Journal of Mathematics, 34, 1239-1254. https://doi.org/10.1216/rmjm/1181069797

[3] Erbe, L. (2001) Oscillation Criteria for Second Order Linear Equations on a Time Scale. Canadian Applied Mathematics Quarterly, 9, 345-375.

[4] Agarwal, R.P., Bohner, M. and Saker, S.H. (2005) Oscillation of Second Order Delay Dynamic Equations. Canadian Applied Mathematics Quarterly, 13, 1-18.

[5] Erbe, L., Peterson, A. and Saker, S.H. (2007) Oscillation Criteria for Second Order Nonlinear Delay Dynamic Equations. Journal of Mathematical Analysis and Applications, 333, 505-522. https://doi.org/10.1016/j.jmaa.2006.10.055

[6] Crace, S.R., Bohner, M. and Agarwal, R.P. (2009) On the Oscillation of Second-Order Half-Linear Dynamic Equations. Journal of Difference Equations and Applications, 15, 451-460. https://doi.org/10.1080/10236190802125371

[7] Crace, S.R., Agarwal, R.P., Kaymakcalan, B. and Sae-jie, W. (2010) Oscillation Theorems for Second Order Nonlinear Dynamic Equations. Journal of Applied Mathematics and Computing, 32, 205-218. https://doi.org/10.1007/s12190-009-0244-7

[8] Sun, S., Han, Z. and Zhang, C. (2009) Oscillation of Second Order Delay Dynamic Equations on Time Scales. Journal of Applied Mathematics and Computing, 30, 459-468. https://doi.org/10.1007/s12190-008-0185-6

[9] Zhang, Q. and Gao, L. (2010) Oscillation Criteria for Second Order Half-Liner Delay Dynamic Equations with Damping on Time Scales. Scientia Sinica Mathematica, 40, 673-682. (In Chinese)

[10] Zhang, Q., Gao, L. and Liu, S. (2011) Oscillation Criteria for Second Order Half-Liner Delay Dynamic Equations with Damping on Time Scales (П). Scientia Sinica Mathematica, 41, 885-896. (In Chinese)

[11] Erbe, L., Peterson, A. and Saker, S.H. (2005) Asymptotic Behavior of Solution of a Third-Order Nonlinear Dynamic Equations. Journal of Computational and Applied Mathematics, 181, 92-102. https://doi.org/10.1016/j.cam.2004.11.021

[12] Erbe, L., Peterson, A. and Saker, S.H. (2007) Hille and Nehari Type Criteria for Third Order Nonlinear Dynamic Equations. Journal of Mathematical Analysis and Applications, 329, 112-131. https://doi.org/10.1016/j.jmaa.2006.06.033

[13] Hassan, T.S. (2009) Oscillation of Third Order Nonlinear Delay Dynamic Equations on Time Scales. Mathematical and Computer Modelling, 49, 1573-1586. https://doi.org/10.1016/j.mcm.2008.12.011

[14] Li, T., Han, Z., Sun, S. and Zhao, Y. (2011) Oscillation Results for Third Order Nonlinear Delay Dynamic Equations on Time Scales. Bulletin of the Malaysian Mathematical Sciences Society, 34, 639-648.

[15] Agarwal, R.P., Bohner, M., Tang, S., Li, T. and Zhang, C. (2012) Oscillation and Asymptotic Behavior of Third-Order Nonlinear Retarded Dynamic Equations. Applied Mathematics and Computation, 219, 3600-3609.

https://doi.org/10.1016/j.amc.2012.09.054

[16] Han, Z., Li, T., Sun, S. and Zhang, M. (2011) Oscillation Behavior of Solution of Third-Order Nonlinear Delay Dynamic Equations on Time Scales. Communica- 
tions of the Korean Mathematical Society, 26, 499-533.

https://doi.org/10.4134/CKMS.2011.26.3.499

[17] Li, T., Han, Z., Sun, S. and Zhao, Y. (2011) Asymptotic Behavior of Solutions for Third-Order Half-Linear Delay Dynamic Equations on Time Scales. Journal of Applied Mathematics and Computing, 36, 333-346.

https://doi.org/10.1007/s12190-010-0406-7

[18] Gao, L., Zhang, Q.X. and Liu, S.H. (2014) Oscillatory Behavior of Third-Order Nonlinear Delay Dynamic Equations on Time Scales. Journal of Computational and Applied Mathematics, 256, 104-113. https://doi.org/10.1016/j.cam.2013.07.024

[19] Bohner, M. and Peterson, A. (2001) Dynamic Equations on Time Scales: An Introduction with Applications. Birkhäuser, Boston.

https://doi.org/10.1007/978-1-4612-0201-1

[20] Agarwal, R.P., Bohner, M., Crace, S.R. and O’Regan, D. (2005) Discrete Oscillation Theory. Hindawi Publishing Corporation, New York.

[21] Hardy, G.H., Littlewood, J.E. and Polya, G. (1988) Inequalities. 2nd Edition, Cambridge University Press, Cambridge.

[22] Tuna, A. and Kutukcu, S. (2008) Some Integral Inequalities on Time Scales. Applied Mathematics and Mechanics, 29, 23-29. https://doi.org/10.1007/s10483-008-0104-y 